\title{
Body weight and 25-hidroxyvitamin D follicular levels: a prospec- tive study of women submitted to in vitro fertilization
}

\author{
Vitor AS Deriquehem ${ }^{1}$, Roberto A Antunes ${ }^{2,3}$, Mila W Reginatto ${ }^{1}$, Ana C Mancebo ${ }^{3}$, Patricia Areas ${ }^{3}$, Enrrico Blo- \\ ise $^{1}$, Maria do Carmo B de Souza ${ }^{3}$, Tania M Ortiga-Carvalho ${ }^{1}$ \\ ${ }^{1}$ Laboratory of Translational Endocrinology, Carlos Chagas Filho Biophysics Institute, Federal University of Rio \\ de Janeiro. \\ ${ }^{2}$ Teaching Maternity Hospital, Federal University of Rio de Janeiro. Rio de Janeiro/RJ, Brazil. \\ ${ }^{3}$ Fertipraxis Clinic, Rio de Janeiro/RJ, Brazil.
}

Parts of this paper were presented in the $19^{\text {th }}$ Annual Congress of the Brazilian Society of Assisted Reproduction.

\begin{abstract}
Objective: Vitamin D deficiency has been largely related to infertility in animals. However, data demonstrating a direct association between hypovitaminosis $D$ and infertility in humans are still conflicting. Increased body weight and an elevated body mass index (BMI) are known for their association with infertility. Therefore, this study attempted to verify whether increases in body weight and the BMI were associated with lower 25-hidroxyvitamin D [25(OH)D3] levels in the follicular fluid (FF) of patients treated for infertility with intracytoplasmic sperm injections (ICSI). This study aimed to assess the FF levels of 25(OH)D3 in women submitted to ICSI and correlate these levels with the different body weight and BMI values observed in the enrolled cohort.

Methods: The FF aspirates of 199 patients submitted to ICSI were collected after oocyte aspiration to check whether FF 25(OH)D3 levels were associated with weight regardless of the etiology of infertility. Chemiluminescent assays were used to assess FF 25(OH)D3 levels. The etiology of infertility was defined based on patient clinical history and follow-up.

Results: The patients enrolled in the study were divided into three groups according to their FF 25(OH)D3 levels, as follows: a) deficient $(\mathrm{n}=71 ;<20 \mathrm{ng} / \mathrm{ml})$; b) insufficient $(\mathrm{n}=64 ; 21<25(\mathrm{OH}) \mathrm{D} 3>29 \mathrm{ng} / \mathrm{ml})$; and c) sufficient $(\mathrm{n}=56>30 \mathrm{ng} / \mathrm{ml})$ levels. Patients with lower FF $25(\mathrm{OH})$ D3 levels had a greater mean weight $(64.1 \mathrm{~kg})$ when compared to patients with higher $25(\mathrm{OH}) \mathrm{D} 3$ levels $(60.7 \mathrm{~kg})$, $p<0.01$. No differences were observed in terms of age or etiology of infertility.

Conclusion: The body weight of the individuals with FF 25(OH)D3 deficiency measured in single follicles was significantly higher regardless of the etiology of infertility. Further epidemiologic and molecular studies are required to verify whether the amount of follicular 25(OH)D3 affects the outcome of IVF procedures.
\end{abstract}

Keywords: Vitamin D, Calcitriol, Follicular fluid, BMI, IVF/ ICSI.

\section{INTRODUCTION}

Vitamin D plays a major role in bone and calcium metabolism, in addition to acting in the regulation of the cardiovascular and immune systems. Humans obtain their daily levels of vitamin $D$ mainly by endogenous biosynthesis induced by exposure to ultraviolet B (UVB) radiation, in concert with a small contribution from the intake of different food sources. Cholecalciferol (vitamin D3) and ergocalciferol (vitamin D2) are hydroxylated in the liver, resulting in 25-hidroxyvitamin $\mathrm{D}$ or $25(\mathrm{OH}) \mathrm{D} 3$. In the kid- neys, $25(\mathrm{OH}) \mathrm{D}$ is metabolized as the active form -calcitriol $(1,25$-dihidroxyvitamin D) - or as an inactive metabolite - 24,25-hidroxyvitamin D. Vitamin D deficiency is defined as serum 25(OH)D3 levels below $20 \mathrm{ng} / \mathrm{ml}$, whereas sufficient levels are defined by values greater than $30 \mathrm{ng} / \mathrm{ml}$ (LeBlanc et al., 2015).

The role of vitamin $D$ in fertility and ovarian function is still largely unexplored. Vitamin D receptor (VDR) expression has been observed in the ovaries of animal models, more specifically in the granulosa cells and follicles (Brannian et al., 2009). There is a direct association between vitamin $D$ levels and fertilization rates in different animal models (Panda et al., 2001; Luk et al., 2012). In humans, the role of vitamin $D$ in fertilization, embryo quality and implantation is still controversial (Terushkin et al., 2010; Aleyasin et al., 2011; Rudick et al., 2012; Aflatoonian et al., 2014; Paffoni et al., 2014; Polyzos et al., 2014). Several authors postulated that implantation and clinical pregnancy rates were lower in patients with serum $25(\mathrm{OH})$ D3 < 20ng/ml (Luk et al., 2012; Paffoni et al., 2014; Perez-Lopez et al., 2015). Better in vitro fertilization (IVF) outcomes were related to increased follicular fluid (FF) levels of 25(OH)D3 (Ozkan et al., 2010; Farzadi et al., 2015). One meta-analysis described an association between lower live birth rates and lower serum 25(OH)D3 levels in women submitted to IVF, with no differences in clinical pregnancy rates (Lv et al., 2016), suggesting that serum 25(OH) D3 was key to pregnancy progression. However, several studies failed to find correlations when clinical pregnancy rates were compared against $25 \mathrm{OH}$ )D3 serum levels in patients submitted to IVF (Aleyasin et al., 2011; Polyzos et al., 2014; Fabris et al., 2014), highliting the need for more studies investigating the impact of $25(\mathrm{OH}) \mathrm{D} 3$ in reproductive outcomes.

In terms of obstetric outcomes, $25(\mathrm{OH}) \mathrm{D} 3$ deficiency has been associated with increased risk of preeclampsia, gestational diabetes, and low birth weight (Aghajafari et al., 2013; Tabesh et al., 2013; Theodoratou et al., 2014).

Body weight and the body mass index (BMI) are important factors associated with hypovitaminosis $D$ and infertility (Wortsman et al., 2000; Reis et al., 2009). A study described a direct association between 25(OH)D3 levels and the BMI, weight, and the waist-hip ratio of a group of women living in the same latitude followed for 30 years. Another study demonstrated that obese and overweight patients had lower 25(OH)D3 levels versus patients with normal weight (Tosunbayraktar et al., 2015). Interestingly, the obese patients had lower levels of $25(\mathrm{OH}) \mathrm{D} 3$ than the overweight individuals. Vitamin $D$ deficiency is highly prevalent among infertile women and has been inversely correlated with the BMI and positively correlated with ex- 
posure to sunlight, but data comparing cause of infertility and height were conflicting. The maternal BMI is also greater in pregnant patients with lower 25(OH)D3 levels.

As for the newborn, umbilical cord blood 25(OH)D3 levels varied according to the season of birth, latitude, and length of the newborn, but not with weight at birth, adiposity, head circumference, or maternal 25(OH)D3 levels. However, maternal 25(OH)D3 levels were positively associated with infant weight at birth related to gestational age. Even though Tian found a positive association between maternal vitamin D levels and infant weight at birth, vitamin $D$ supplementation for pregnant women with low serum levels of $25(\mathrm{OH}) \mathrm{D} 3$ failed to improve birth weight outcomes. In fact, the benefits associated with gestational vitamin D supplementation are debatable, and more research is warranted (Dalgard et al., 2016; Dressler et al., 2016).

In a nutshell, it is still fairly unclear whether FF levels of $25(\mathrm{OH}) \mathrm{D} 3$ are associated with the BMI or the weight of infertile women, and if infertility can be correlated at all with $25(\mathrm{OH}) \mathrm{D} 3$ levels in the FF. Therefore, this study aimed to assess whether there are similarities in the serum and follicular levels of 25(OH)D and to analyze the FF levels of 25(OH)D3 in women with different BMIs submitted to ICSI.

\section{MATERIALS AND METHODS Patients}

The patients enrolled in this study underwent ICSI at the Fertipraxis Center for Human Reproduction, a clinic certified by the Brazilian health surveillance authority (ANVISA) and the Latin American Network of Assisted Reproduction (REDLARA). The local Ethics Committee approved the study protocol; all enrolled patients gave written consent before joining the study.

\section{Follicular fluid aspiration}

All female patients referred to oocyte aspiration between July of 2012 and November of 2014 had a sample of follicular fluid collected on the same day of oocyte pickup. The samples were collected using a sterile vacuum system set to $90 \mathrm{mmHg}$ with a $17 \mathrm{G}$ Wallace $\AA$ aspiration needle. In order to obtain a pure, single follicular fluid sample, without the contamination of blood or fluid from other follicles during aspiration, the aspiration procedure was initiated in the first follicle measuring more than $17 \mathrm{~mm}$. As soon as the follicle was emptied, the aspiration needle was removed from the ovary and all the fluid content that was still in the circuit of the vacuum system was transferred to a sterile storage container. Then, the container with the sample was disconnected from the vacuum system and taken to the lab. At the lab, an embryologist searched the fluid to retrieve its oocyte and, after the oocyte was removed, the follicular fluid was placed in a sterile cryo tube and immediately frozen in liquid nitrogen. A new storage container was then connected to the vacuum system and the oocyte aspiration resumed, this time pooling all the remaining follicular fluid aspirated in order to retrieve as many oocytes as possible. For purposes of analysis, all samples were thawed and centrifuged at $1500 \mathrm{~g}$ for 15 minutes. Patients submitted to ovarian hyper stimulation protocols with aromatase inhibitors were excluded. The included individuals were on protocols with either recombinant FSH (fresh) or $r$ FSH plus purified HMG (Urinary Menopausal Gonadotropins, us + lush) associated to GnRH antagonists.

\section{Vitamin D level measurements}

FF 25(OH)D3 levels were assessed with a chemiluminescent immunoassay (Elecsys total vitamin D -Roche Diagnostics, Brazil) at the Laboratory of Molecular and Trans- lational Endocrinology, Department of Medicine, Paulista School of Medicine, Federal University of São Paulo (Unifesp/EPM), São Paulo, Brazil. Inter-assay and intra-assay variation was $8.3 \%$ and $9.1 \%$, respectively.

\section{BMI calculation}

The BMI was calculated based on the height and weight measured for each patient. The causes of infertility were described in the patients' charts.

\section{Statistics}

The data were presented in the form of mean \pm standard deviation. The D'Agostino-Pearson test was used to test for normality. One-way analysis of variance (ANOVA) and the Bonferroni multiple comparisons test were used in the analysis of body weight, BMI, age, and 25(OH)D3 levels one. The Bland-Altman plot and Pearson's correlation coefficient were used to validate the association between serum and FF 25(OH)D3 levels. Statistical analyses were performed using the Graphpad Prism 6 Software (GraphPad Software, Inc., San Diego, CA, USA). Significance was attributed to differences with a $P<0.05$.

\section{RESULTS}

One hundred and ninety-nine patients were enrolled in this study. Figure 1 shows strong positive correlations between the serum and FF 25(OH)D3 levels of 14 randomly selected patients. The Bland-Altman plot showed a bias close to zero $(0.12)$, confirming the strength of the correlation.

Figure 2 shows the patient BMIs sorted into groups based on 25(OH)D3 FF levels. Four percent of the patients had low BMIs ( $<18.5 \mathrm{~kg} / \mathrm{m} 2), 67 \%$ had normal BMIs (20 to $25 \mathrm{~kg} / \mathrm{m} 2$ ), and $19 \%$ were overweight or obese $(>25 \mathrm{~kg} /$ $\mathrm{m} 2)$. Patients with lower levels of $25(\mathrm{OH}) \mathrm{D} 3(<20 \mathrm{nl} /$ $\mathrm{ml})$ had higher BMIs $(P<0.03)$. When the patients were sorted based on their BMIs, the group with the higher BMIs $(>25 \mathrm{~kg} / \mathrm{m} 2)$ had lower levels of FF $25(\mathrm{OH}) \mathrm{D} 3$. The patients were then divided into two groups according to whether they had FF levels of $25(\mathrm{OH}) \mathrm{D} 3$ lower or higher than $20 \mathrm{ng} / \mathrm{ml}$.

The patients enrolled in the study had a mean age of $35.9 \pm 4.2$ years. The youngest was 22 and the oldest 48 years old. Seventy-one women had 25(OH)D3 follicular fluid levels below $20 \mathrm{ng} / \mathrm{ml}$, with a mean value of $13.2 \pm 4.1$ $\mathrm{ng} / \mathrm{ml}$, and 128 women had follicular fluid levels of $25(\mathrm{OH})$ D3 above $20 \mathrm{ng} / \mathrm{ml}$, with a mean value of $30.7 \pm 8.3 \mathrm{ng} /$ $\mathrm{ml}$. No differences were observed in the age and total doses of recombinant FSH given to both groups. The FF $25(\mathrm{OH}) \mathrm{D} 3$ levels seen in both groups were significantly different $(P<0.05)$. The causes of infertility were distributed almost equally $(P>0.05)$, with unexplained reasons and reduced ovarian reserve ranking as the most prevalent ( $35 \%$ vs. $33.5 \%)$, followed by male factor infertility $(30 \%$ vs. $24.2 \%)$, tubal factor infertility or endometriosis (20\% vs. $15.6 \%)$, mixed factors $(7.5 \%$ vs. $7.8 \%)$, and others $(7.5 \%$ vs. $18.9 \%)$. Patients with FF $25(\mathrm{OH})$ D3 deficiency were heavier than their counterparts with higher levels of vitamin D $(64.1 \pm 12.1 \mathrm{~kg}$ vs. $60.7 \pm 10.5 \mathrm{~kg}, P<0.01$, Table 1 and Figure 3 ).

\section{DISCUSSION}

This study looked into a cohort of Brazilian infertile women to see whether their FF $25(\mathrm{OH}) \mathrm{D} 3$ levels would vary depending on their weights and BMIs. The complex ethnic makeup of the group prevented the description of differences based on ethnicity. Therefore, the patients were divided into groups having $25(\mathrm{OH}) \mathrm{D} 3$ follicular levels as basis. First we checked whether follicular vitamin D levels reproduced serum vitamin D levels. Few studies 
Table 1. Weight, age, recombinant FSH doses and causes of infertility

\begin{tabular}{|c|c|c|c|}
\hline & $\begin{array}{c}25 \mathrm{OH} \mathrm{D3}(\mathrm{ng} / \mathrm{ml}) \\
<20\end{array}$ & $\begin{array}{c}25 \mathrm{OH} \mathrm{D3}(\mathrm{ng} / \mathrm{ml}) \\
>20\end{array}$ & $P$ \\
\hline $\mathrm{n}$ & 71 & 128 & \\
\hline Weight $(\mathrm{Kg})$ & $64.1 \mathrm{~kg} \pm 12.1$ & $60.7 \mathrm{~kg} \pm 10.5$ & $<0.05$ \\
\hline Age (years) & 36.3 & 35.6 & $>0.05$ \\
\hline \multicolumn{4}{|l|}{ Causes of Infertility (\%) } \\
\hline Unexplained/reduced ovarian reserve & 35 & 33.5 & $>0.05$ \\
\hline Male factor infertility & 30 & 24.2 & $>0.05$ \\
\hline Tubal factor infertility/endometriosis & 20 & 15.6 & $>0.05$ \\
\hline Mixed & 7.5 & 7.8 & $>0.05$ \\
\hline Others & 7.5 & 18.9 & $>0.05$ \\
\hline
\end{tabular}

Figure 1. Follicular and Serum $25(\mathrm{OH})$ levels of 14 patients. Pearson's correlation coefficient and Bland-Altman plots.

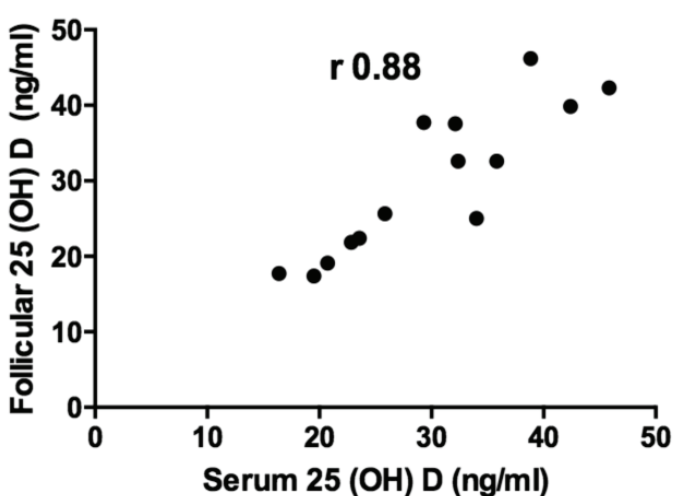

Figure 2. Body mass index $(\mathrm{m} 2 / \mathrm{kg})$ and follicular levels of $25(\mathrm{OH})$ D3 $(\mathrm{ng} / \mathrm{ml})$. Individual patients are shown. Lines represent mean values and standard deviation.

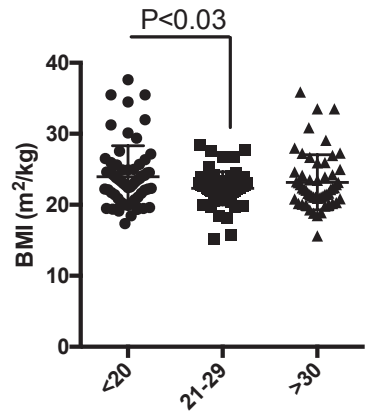

$25(\mathrm{OH}) \mathrm{D} 3(\mathrm{ng} / \mathrm{ml})$

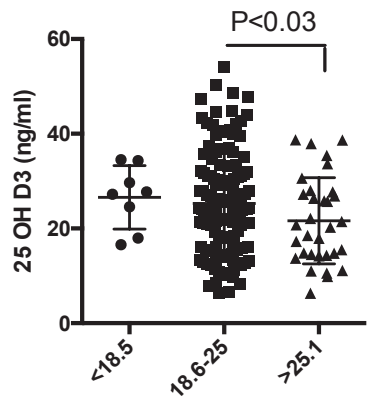

$\mathrm{BMI}\left(\mathrm{m}^{2} / \mathrm{kg}\right)$
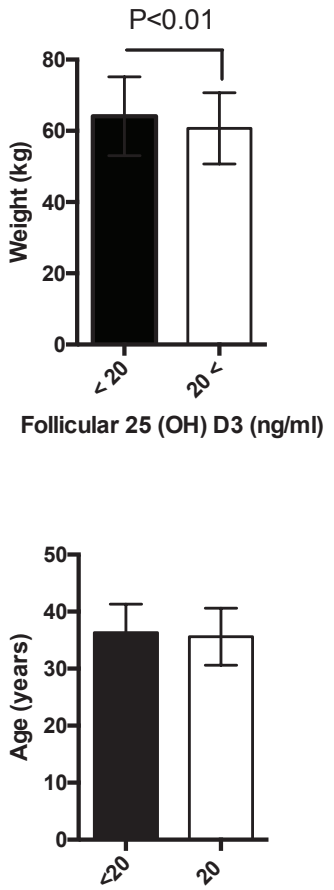

Follicular 25 (OH) D3 (ng/ml)

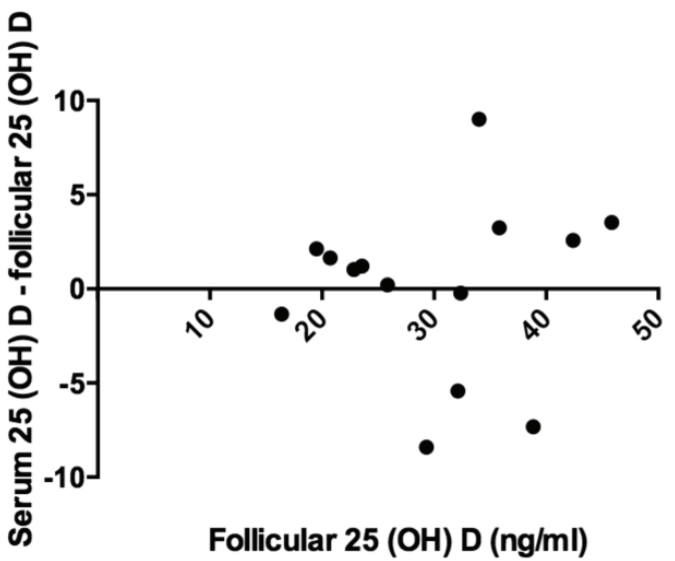

Figure 3. Weight $(\mathrm{kg})$ and age (years) of patients with $>20 \mathrm{ng} / \mathrm{ml}$ and $<20 \mathrm{ng} / \mathrm{ml}$ of $25(\mathrm{OH})$ D3. Bars represent mean values and standard deviation.

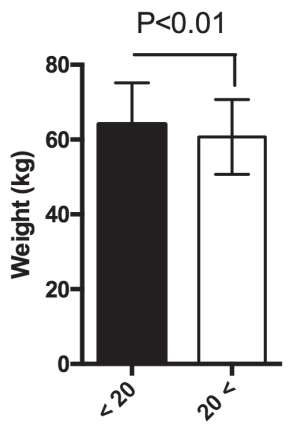

Follicular $25(\mathrm{OH}) \mathrm{D} 3(\mathrm{ng} / \mathrm{ml})$

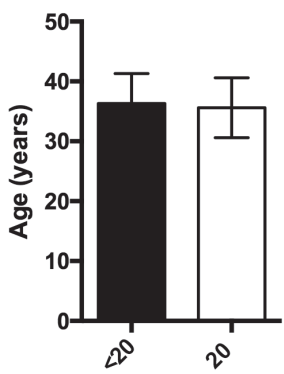

Follicular $25(\mathrm{OH}) \mathrm{D} 3(\mathrm{ng} / \mathrm{ml})$ 
described an equivalence between follicular fluid (FF) and serum levels of 25(OH)D3 (Ozkan et al., 2010; Aleyasin et al., 2011; Rudick et al., 2012; Firouzabadi et al., 2014). Data analysis by Pearson's correlation coefficient and Bland-Altman plots confirmed the existence of such equivalence, and follicular levels were then used to stratify patients in this study.

The American Society of Endocrinology categorizes subjects based on their 25(OH)D3 levels: 20, 21-29, and $30 \mathrm{ng} / \mathrm{ml}$ are the cutoffs for deficiency, insufficiency and sufficiency, respectively (Holick et al., 2011). The correlation between 25(OH)D3 deficiency and obesity has been reported (Wortsman et al., 2000; Harel et al., 2011). In our cohort, most of the subjects had normal body weights and BMIs of less than $25 \mathrm{~m} 2 / \mathrm{kg}$; decreases in follicular 25(OH)D3 levels were observed among overweight and obese individuals ( $>25.1 \mathrm{~m} 2 / \mathrm{kg}$ ). Obesity is known to affect male and female fertility, and many authors have explored this subject (Brannian et al., 2009; Dressler et al., 2016). However, it is difficult to separate the effects of obesity alone from factors associated to sedentarism and hormone levels altered by weight gain.

Controversy still looms over the categorization of $25(\mathrm{OH}) \mathrm{D} 3$ levels as sufficient or insufficient. The capture of 25(OH)D3 by adipocytes or the idea that adipocytes act as a reservoir of calcitriol may be true; yet, the two theories still lack supporting evidence. Patients presenting with $25(\mathrm{OH}) \mathrm{D} 3$ serum levels of less than $20 \mathrm{ng} / \mathrm{ml}$ are currently considered deficient. The groups in this study were divided based on this threshold, and each was evaluated for their differences. The age and prescribed dose of recombinant FSH were not different, but patients with $25(\mathrm{OH}) \mathrm{D} 3$ deficiency were heavier than the individuals with levels greater than $20 \mathrm{ng} / \mathrm{ml}$. The association between weight and FF levels of 25(OH)D3 may be affected by multiple factors, such as a sedentary lifestyle and/or a poor diet. Another plausible explanation is the molecular abduction of vitamin $\mathrm{D}$ by adipose tissue discussed above. One of the strengths of our study is the fact that each sample corresponds to one pre-ovulatory follicle from each woman. However, the study's limitations include the small number subjects enrolled and the lack of a lifestyle assessment questionnaire.

The two groups were also analyzed for infertility. Unexplained infertility and reduced ovarian reserve were the main reported causes of infertility, followed by male factor infertility and tubal factor infertility/endometriosis. Patients with more than one factor were classified as "Mixed," since a single factor could not be directly associated with the observed follicular levels of $25(\mathrm{OH}) \mathrm{D} 3$. Both groups had equal distributions of causes of infertility, suggesting the absence of an association with the metabolism of vitamin $D$.

To sum up with, women with deficient levels of $25(\mathrm{OH})$ D3 measured in single follicles were significantly heavier regardless of their infertility statuses. The level of follicular 25(OH)D3 within individual follicles may possibly impact the outcome of IVF procedures. However, further epidemiologic and molecular studies are warranted to better understand the impact of FF 25(OH)D3 levels in reproduction.

\section{Acknowledgements}

The authors would like to thank the staff of the Laboratory of Molecular and Translational Endocrinology, Department of Medicine, Paulista School of Medicine, Federal University of São Paulo (Unifesp/EPM), São Paulo, Brazil, FF assays, for their invaluable support. Grants and fellowships: CNPq/ PIBIC to VDAS; CNPq to TMO-C and EB, FAPERJ to TMOC and CAPES to MWR.

Funding: this study was funded by the FAPERJ and CNPq (Grant: to T.M.O- C.).

\section{CONFLICT OF INTERESTS}

No conflict of interest have been declared.

\section{Corresponding author:}

Tania M. Ortiga-Carvalho

Laboratory of Translational Endocrinology

Instituto de Biofísica Carlos Chagas Filho

Federal University of Rio de Janeiro

Cidade Universitária Rio de Janeiro/RJ, Brasil

E-mail: taniaort@biof.ufrj.br

\section{REFERENCES}

Aflatoonian A, Arabjahvani F, Eftekhar M, Sayadi M. Effect of vitamin $D$ insufficiency treatment on fertility outcomes in frozen-thawed embryo transfer cycles: A randomized clinical trial. Iran J Reprod Med. 2014; 12:595-600.

Aghajafari F, Nagulesapillai T, Ronksley PE, Tough SC, O'Beirne M, Rabi DM. Association between maternal serum 25-hydroxyvitamin D level and pregnancy and neonatal outcomes: systematic review and meta-analysis of observational studies. BMJ. 2013; 346:f1169.

Aleyasin A, Hosseini MA, Mahdavi A, Safdarian L, Fallahi $P$, Mohajeri MR, Abbasi M, Esfahani F. Predictive value of the level of vitamin $D$ in follicular fluid on the outcome of assisted reproductive technology. Eur J Obstet Gynecol Reprod Biol. 2011; 159:132-7.

Brannian J, Eyster K, Greenway M, Henriksen C, Teslaa K, Diggins M. Progressive obesity leads to altered ovarian gene expression in the Lethal Yellow mouse: a microarray study. J Ovarian Res. 2009; 2:10.

Dalgard C, Petersen MS, Steuerwald U, Weihe P, Grandjean P. Umbilical Cord Serum 25-Hydroxyvitamin D Concentrations and Relation to Birthweight, Head Circumference and Infant Length at Age 14 Days. Paediatr Perinat Epidemiol. 2016; 30: 238-45.

Dressler N, Chandra A, Aguirre Davila L, Spineli LM, Schippert C, von Versen-Hoynck F. BMI and season are associated with vitamin $D$ deficiency in women with impaired fertility: a two-centre analysis. Arch Gynecol Obstet. 2016; 293: $907-14$.

Fabris A, Pacheco A, Cruz M, Puente JM, Fatemi H, Garcia-Velasco JA. Impact of circulating levels of total and bioavailable serum vitamin $D$ on pregnancy rate in egg donation recipients. Fertil Steril. 2014; 102: 1608-12.

Farzadi L, Khayatzadeh Bidgoli H, Ghojazadeh M, Bahrami Z, Fattahi A, Latifi Z, Shahnazi V, Nouri M. Correlation between follicular fluid $25-\mathrm{OH}$ vitamin $\mathrm{D}$ and assisted reproductive outcomes. Iran J Reprod Med. 2015; 13: 361-6.

Firouzabadi RD, Rahmani E, Rahsepar M, Firouzabadi MM. Value of follicular fluid vitamin $D$ in predicting the pregnancy rate in an IVF program. Arch Gynecol Obstet. 2014; 289: 201-6.

Harel Z, Flanagan P, Forcier M, Harel D. Low vitamin D status among obese adolescents: prevalence and response to treatment. J Adolesc Health. 2011; 48: 448-52.

Holick MF, Binkley NC, Bischoff-Ferrari HA, Gordon CM, Hanley DA, Heaney RP, Murad MH, Weaver CM; Endocrine Society. Evaluation, treatment, and prevention of vitamin D deficiency: an Endocrine Society clinical 
practice guideline. J Clin Endocrinol Metab. 2011; 96: 1911-30.

LeBlanc ES, Zakher B, Daeges M, Pappas M, Chou R. Screening for vitamin $D$ deficiency: a systematic review for the U.S. Preventive Services Task Force. Ann Intern Med. 2015; 162: 109-22.

Luk J, Torrealday S, Neal Perry G, Pal L. Relevance of vitamin D in reproduction. Hum Reprod. 2012; 27: 3015-27.

Lv SS, Wang JY, Wang XQ, Wang Y, Xu Y. Serum vitamin $D$ status and in vitro fertilization outcomes: a systematic review and meta-analysis. Arch Gynecol Obstet. 2016; 293:1339-45.

Ozkan S, Jindal S, Greenseid K, Shu J, Zeitlian G, Hickmon C, Pal L. Replete vitamin D stores predict reproductive success following in vitro fertilization. Fertil Steril. 2010; 94: 1314-9.

Paffoni A, Ferrari S, Vigano P, Pagliardini L, Papaleo E, Candiani M, Tirelli A, Fedele L, Somigliana E. Vitamin D deficiency and infertility: insights from in vitro fertilization cycles. J Clin Endocrinol Metab. 2014; 99: E2372-6.

Panda DK, Miao D, Tremblay ML, Sirois J, Farookhi R, Hendy GN, Goltzman D. Targeted ablation of the 25-hydroxyvitamin D 1alpha -hydroxylase enzyme: evidence for skeletal, reproductive, and immune dysfunction. Proc Natl Acad Sci U S A. 2001; 98: 7498-503.

Perez-Lopez FR, Pasupuleti $V$, Mezones-Holguin E, Benites-Zapata VA, Thota P, Deshpande A, Hernandez AV. Effect of vitamin $D$ supplementation during pregnancy on maternal and neonatal outcomes: a systematic review and meta-analysis of randomized controlled trials. Fertil Steril. 2015; 103: 1278-88e4.
Polyzos NP, Anckaert E, Guzman L, Schiettecatte J, Van Landuyt L, Camus M, Smitz J, Tournaye H. Vitamin $D$ deficiency and pregnancy rates in women undergoing single embryo, blastocyst stage, transfer (SET) for IVF/ICSI. Hum Reprod. 2014; 29: 2032-40.

Reis JP, von Muhlen D, Miller ER 3rd, Michos ED, Appel LJ. Vitamin D status and cardiometabolic risk factors in the United States adolescent population. Pediatrics. 2009; 124: e371-9.

Rudick B, Ingles S, Chung K, Stanczyk F, Paulson R, Bendikson K. Characterizing the influence of vitamin D levels on IVF outcomes. Hum Reprod. 2012; 27: 3321-7.

Tabesh M, Salehi-Abargouei A, Tabesh M, Esmaillzadeh A. Maternal vitamin D status and risk of pre-eclampsia: a systematic review and meta-analysis. J Clin Endocrinol Metab. 2013; 98: 3165-73.

Terushkin V, Bender A, Psaty EL, Engelsen O, Wang SQ, Halpern AC. Estimated equivalency of vitamin D production from natural sun exposure versus oral vitamin $D$ supplementation across seasons at two US latitudes. J Am Acad Dermatol. 2010; 62: 929.e1-9.

Theodoratou E, Tzoulaki I, Zgaga L, Ioannidis JP. Vitamin $D$ and multiple health outcomes: umbrella review of systematic reviews and meta-analyses of observational studies and randomised trials. BMJ. 2014; 348: g2035.

Wortsman J, Matsuoka LY, Chen TC, Lu Z, Holick MF. Decreased bioavailability of vitamin $\mathrm{D}$ in obesity. Am J Clin Nutr. 2000; 72: 690-3. 